\title{
Fit of electroweak parameters in polarised deep-inelastic scattering using data from the $\mathrm{H} 1$ experiment
}

\section{Daniel Britzger*i}

DESY, Notkestr. 85, 22607 Hamburg, Germany

E-mail: daniel.britzger@desy.de

\begin{abstract}
Using the final deep-inelastic $e^{+} p$ and $e^{-} p$ neutral and charged current scattering cross sections from the H1 experiment at the HERA collider the vector and axial-vector couplings $v_{q}$ and $a_{q}$ of light quarks $u$ and $d$ to the $Z^{0}$-boson are determined [1]. The uncertainties on the parton distributions functions (PDFs) are accounted for by simultaneously fitting the electroweak parameters and the parameters of the PDFs. By using data with polarised electron beams in the fit, the precision is improved compared to an earlier analysis. The combined QCD and electroweak analysis is employed for a consistency study of the Standard Model of Particle Physics in the domain of space-like momentum transfer by determining the masses of the $Z^{0}$ and $W$-bosons, and by determining the Fermi coupling constant $G_{\mathrm{F}}$ or the weak mixing angle $\sin ^{2} \theta_{W}$ together with the $W$-boson mass. The weak mixing angle, defined in the on-shell scheme, is determined simultaneously at various scales in the range of the data of $12<Q^{2}<50000 \mathrm{GeV}^{2}$ and thus probes the weak interaction at various scales from one process. All results are found to be compatible with the Standard Model expectations or with the currently most precise values from other data.
\end{abstract}

XXIV International Workshop on Deep-Inelastic Scattering and Related Subjects

11-15 April, 2016

DESY Hamburg, Germany

\footnotetext{
*Speaker.

${ }^{\dagger}$ for the H1 Collaboration and H. Spiesberger
} 


\section{Introduction}

The H1 experiment at the HERA collider recorded collisions of electrons (or positrons) of $27.6 \mathrm{GeV}$ and protons of up to $920 \mathrm{GeV}$ and thus provides a large set of cross sections for neutral and charged current deep-inelastic scattering (NC and CC DIS). These NC and CC cross sections are important ingredients for the determination of parton distribution functions of the proton (PDFs), which are indispensible ingredients for precision measurements and searches for physics beyond the Standard Model (SM) at hadron colliders, such as the LHC. The H1 data on NC and CC scattering has already been combined with data from the ZEUS experiments and the extraction of the PDFs was studied [2].

In this report, we focus on precision studies of electroweak parameters involved in the interaction using published $\mathrm{NC}$ and $\mathrm{CC}$ cross sections from the $\mathrm{H} 1$ experiment. At HERA, a first determination of the weak neutral couplings of the light quarks to the $Z^{0}$-boson, the axial coupling, $a_{q}$, and the vector-axial coupling, $v_{q}$, was performed using $\mathrm{H} 1$ data taken during the first phase of the HERA operation (HERA-I) [3]. These studies are extended and all the H1 data on NC and CC DIS are included in the determination, where in particular the HERA-II data taken with longitudinally polarised lepton beams at higher values of $Q^{2}\left(150<Q^{2}<50000 \mathrm{GeV}^{2}\right)$ improve the precision compared to the extraction based on HERA-I data alone. The quark couplings are extracted in a combined QCD and electroweak fit in order to account for the uncertainties on the PDFs on the electroweak parameters. The analysis is extended to an extraction of other parameters of the SM, such as the masses of the $Z$ and $W$-boson, the weak mixing angle $\sin ^{2} \theta_{W}$ or the Fermi coupling constant $G_{\mathrm{F}}$. The space-like momentum transfer in DIS allows for a unique test of the SM complementary to direct measurements at the Z-pole. A similar study was performed by the ZEUS collaboration taking $\mathrm{H} 1$ and ZEUS data into account in their fits [4].

\section{Fit methodology}

NC interactions ${ }^{1}$ in the process $e^{ \pm} p \rightarrow e^{ \pm} X$ are mediated by a virtual photon $(\gamma)$ or $Z^{0}$ boson in the $t$-channel and the cross sections can be expressed in terms of generalised structure functions $\tilde{F}_{2}^{ \pm}, x \tilde{F}_{3}^{ \pm}$and $\tilde{F}_{\mathrm{L}}^{ \pm}$as

$$
\frac{d^{2} \sigma^{\mathrm{NC}}\left(e^{ \pm} p\right)}{d x d Q^{2}}=\frac{2 \pi \alpha^{2}}{x Q^{2}}\left[Y_{+} \tilde{F}_{2}^{ \pm}\left(x, Q^{2}\right) \mp Y_{-} x \tilde{F}_{3}^{ \pm}\left(x, Q^{2}\right)-y^{2} \tilde{F}_{\mathrm{L}}^{ \pm}\left(x, Q^{2}\right)\right],
$$

where $\alpha$ is the fine structure constant at $Q^{2}=0 \mathrm{GeV}^{2}$. The helicity dependence of the interactions are contained in the terms $Y_{\mp}=1 \pm(1-y)^{2}$ with $y$ being the inelasticity of the process. The generalised structure functions are further expressed separately for pure photon or $Z$-exchange or for the interference of those two as

$$
\begin{aligned}
\tilde{F}_{2}^{ \pm} & =F_{2}-\left(v_{e} \pm P_{e} a_{e}\right) \kappa_{Z} F_{2}^{\gamma Z}+\left[\left(v_{e}^{2}+a_{e}^{2}\right) \pm 2 P_{Z} a_{e}\right] \kappa_{Z}^{2} F_{2}^{Z} \\
x \tilde{F}_{3}^{ \pm} & =-\left(a_{e} \pm P_{e} v_{e}\right) \kappa_{Z} x F_{3}^{\gamma Z}+\left[2 v_{e} a_{e} \pm P_{e}\left(v_{e}^{2}+a_{e}^{2}\right)\right] \kappa_{Z}^{2} F_{3}^{Z},
\end{aligned}
$$

\footnotetext{
${ }^{1}$ Here, we focus on the description of the NC process due to its importance for the extraction of the weak neutral couplings. The CC process can be expressed similarly as the NC process in terms of structure functions and is important for the extractions of the $W$-boson mass. More details can be found elsewhere [5].
} 
and similarly for $\tilde{F}_{L}$, where the term $\kappa_{Z}$ accounts for $Z$-exchange. It is expressed in the on-shell scheme as

$$
\kappa_{Z}\left(Q^{2}\right)=\frac{Q^{2}}{Q^{2}+m_{Z}^{2}} \frac{G_{\mathrm{F}} m_{Z}^{2}}{2 \sqrt{2} \pi \alpha}
$$

with the Fermi coupling constant calcuated from the boson and fermion masses as

$$
G_{\mathrm{F}}=\frac{2 \pi \alpha}{2 \sqrt{2} m_{W}^{2}}\left(1-\frac{m_{W}^{2}}{m_{Z}^{2}}\right)^{-1}(1+\Delta r) .
$$

The term $\Delta r=\Delta r\left(\alpha, m_{W}, m_{Z}, m_{t}, m_{H}, \ldots\right)$ contains corrections to the muon decay and is calculated from the fine structure constant and the boson and fermion masses using the program EPRC [6]. The structure functions are related to linear combinations of the quark and anti-quark momentum distributions, $x q$ and $x \bar{q}$, and read for instance for the $F_{2}$ and $x F_{3}$ structure functions in the quarkparton model as:

$$
\begin{aligned}
{\left[F_{2}, F_{2}^{\gamma Z}, F_{2}^{Z}\right] } & =x \sum_{q}\left[e_{q}^{2}, 2 e_{q} v_{q}, v_{q}^{2}+a_{q}^{2}\right]\{q+\bar{q}\}, \\
{\left[F_{3}^{\gamma Z}, F_{3}^{Z}\right] } & =x \sum_{q}\left[2 e_{q} a_{q}, 2 v_{q} a_{q}\right]\{q+\bar{q}\},
\end{aligned}
$$

where $a_{q}$ and $v_{q}$ are the weak neutral axial and vector-axial couplings of the quarks to the Z-boson. The couplings are predicted by the SM to be $a_{q}=I_{q, L}^{(3)}$ and $v_{q}=I_{q, L}^{(3)}-2 e_{q} \sin ^{2} \theta_{W}$, with $I_{q, L}^{(3)}$ being the third component of the left-handed isospin, or more generally those parameters may be considered as individual parameters to the calculations. Higher-order weak corrections may be taken into account in terms of form-factors, but these corrections are small and only considered as corrections $\Delta r$ to the muon decay constant $G_{\mathrm{F}}$ and are not considered otherwise.

The quark couplings of the $u$ and $d$-type quarks $a_{u, d}$ and $v_{u, d}$ are determined in a combined fit together with the parameters of the PDFs. The parameterisation and calculations of the PDFs follows closely the methodology as outlined in ref. [2], where we use here calculations in the zeromass variable-flavor-number-scheme (ZM-VFNS) in NNLO accuracy using the program QCDNUM [7] and the data are restricted to $Q^{2} \geq 12 \mathrm{GeV}^{2}$. The goodness of fit quantity, which is subject to the Minuit minimisation algorithm, is derived from a likelihood function assuming the probability densities to be log-normal distributed:

$$
\chi^{2}=\sum_{i j}\left(\log \left(d_{i}\right)-\log \left(t_{i}\right)\right) V_{i j}^{-1}\left(\log \left(d_{j}\right)-\log \left(t_{j}\right)\right),
$$

where the sum runs over all data points and $d$ and $t$ denote the data and the predictions, respectively, and the covariance matrix $V$ is constructed from all relative uncertainties, taking also correlations between the data sets into account.

All published $\mathrm{NC}$ and $\mathrm{CC}$ cross sections from $\mathrm{H} 1$ are input to the fit and are summarised in table 1 . The cross sections for lower values of $Q^{2}$ at $Q^{2}<150 \mathrm{GeV}^{2}$ have already been combined into a single data set. Cross sections at high- $Q^{2}$ are separately available for the individual running periods, where the data during HERA-II has been taken with longitudinally polarised lepton beams, which improves the sensitivity to $v_{q}$. The beam polarisations are free parameters in the fit and are constrained by taking the measurements of the polarisation as additional data points in the calculation of $\chi^{2}$. 


\begin{tabular}{|c|c|c|c|c|c|}
\hline Dataset & $\begin{array}{l}Q^{2} \text {-range } \\
{\left[\mathrm{GeV}^{2}\right]}\end{array}$ & $\begin{array}{c}\sqrt{s} \\
{[\mathrm{GeV}]}\end{array}$ & No. of datapoints & $\begin{array}{c}\text { Polarisation } \\
{[\%]}\end{array}$ & Ref. \\
\hline$e^{+}$Combined low- $Q^{2}$ & $(0.5) 12-150$ & 319 & $81(262)$ & - & [8] \\
\hline$e^{+}$Combined low $-E_{p}$ & (1.5) $12-90$ & 301 & $118(136)$ & - & [8] \\
\hline$e^{+} \mathrm{NC} 94-97$ & $150-30000$ & 301 & 130 & - & [9] \\
\hline$e^{+}$CC 94-97 & $300-15000$ & 301 & 25 & - & [9] \\
\hline$e^{-}$NC 98-99 & $150-30000$ & 319 & 126 & - & {$[10]$} \\
\hline$e^{-}$CC 98-99 & $300-15000$ & 319 & 28 & - & [10] \\
\hline$e^{-}$NC 98-99 high-y & $100-800$ & 319 & 13 & - & {$[11]$} \\
\hline$e^{+}$NC 99-00 & $150-30000$ & 319 & 147 & - & {$[11]$} \\
\hline$e^{+}$CC 99-00 & $300-15000$ & 319 & 28 & - & {$[11]$} \\
\hline$e^{+}$NC L HERA-II & $120-30000$ & 319 & 137 & $-37.0 \pm 1.0$ & {$[12]$} \\
\hline$e^{+}$CC L HERA-II & $300-15000$ & 319 & 28 & $-37.0 \pm 1.0$ & {$[12]$} \\
\hline$e^{+}$NC R HERA-II & $120-30000$ & 319 & 137 & $+32.5 \pm 0.7$ & {$[12]$} \\
\hline$e^{+}$CC R HERA-II & $300-15000$ & 319 & 28 & $+32.5 \pm 0.7$ & {$[12]$} \\
\hline$e^{-}$NC L HERA-II & $120-50000$ & 319 & 138 & $-25.8 \pm 0.7$ & {$[12]$} \\
\hline$e^{-}$CC L HERA-II & $300-30000$ & 319 & 29 & $-25.8 \pm 0.7$ & {$[12]$} \\
\hline$e^{-}$NC R HERA-II & $120-30000$ & 319 & 139 & $+36.0 \pm 0.7$ & {$[12]$} \\
\hline$e^{-}$CC R HERA-II & $300-15000$ & 319 & 28 & $+36.0 \pm 0.7$ & {$[12]$} \\
\hline
\end{tabular}

Table 1: Datasets used in the combined electroweak and QCD fit. The low and medium $Q^{2}$ datasets are combined data samples from all the measurements published by H1. For each of the datasets, the corresponding range in $Q^{2}$, the center-of-mass energy $\sqrt{s}$, the number of measured datapoints, and the average polarisation value are given. Data taken during the HERA-I running period are taken with unpolarised lepton beams. The numbers in brackets denote the respective quantities for the full dataset, i.e. without the cut of $Q^{2} \geq 12 \mathrm{GeV}^{2}$ applied.

\section{Results}

The fits are performed using the Alpos fitting framework and yield a quality of $\chi^{2} / n_{\text {dof }}=$ $1370.5 /(1388-21)$, where the degrees of freedom is calculated from 1384 cross section data points, 4 polarisation values, 13 PDF parameters, 4 quark copuling parameters and 4 polarisation values. The results of the fit are shown in fig. 1 and are consistent with the SM expectations within the $68 \%$ confidence level. Compared to the extractions from HERA-I data alone, the precision of the vector-axial couplings has been improved significantly. If the couplings of the $u$ and $d$-type quarks are extracted separately, their uncertainties reduce significantly because the results of the quark couplings are correlated. The results are also consistent and with comparable precision than extractions from other processes. The results are found to be compatible with fits where alternatively external PDFs, such as CT10, MSTW or NNPDF3.0, were used.

The NC and CC processes provide a unique opportunity to test the parameters of the SM in the domain of space-like momentum transfers. The fit of the masses of the $Z$ and $W$-boson $\left(m_{Z}\right.$ and $m_{W}$ ), together with the PDFs, are displayed in fig. 2 . The results are compatible with the bestknown values [5] within a confidence level of $68 \%$. Taking $m_{Z}$ as external parameter to the fit [5], the value of $m_{W}$ is determined to

$$
m_{W}=80.407 \pm 0.118(\exp , \mathrm{pdf}) \pm 0.005\left(m_{Z}, m_{t}, m_{H}\right) \mathrm{GeV},
$$



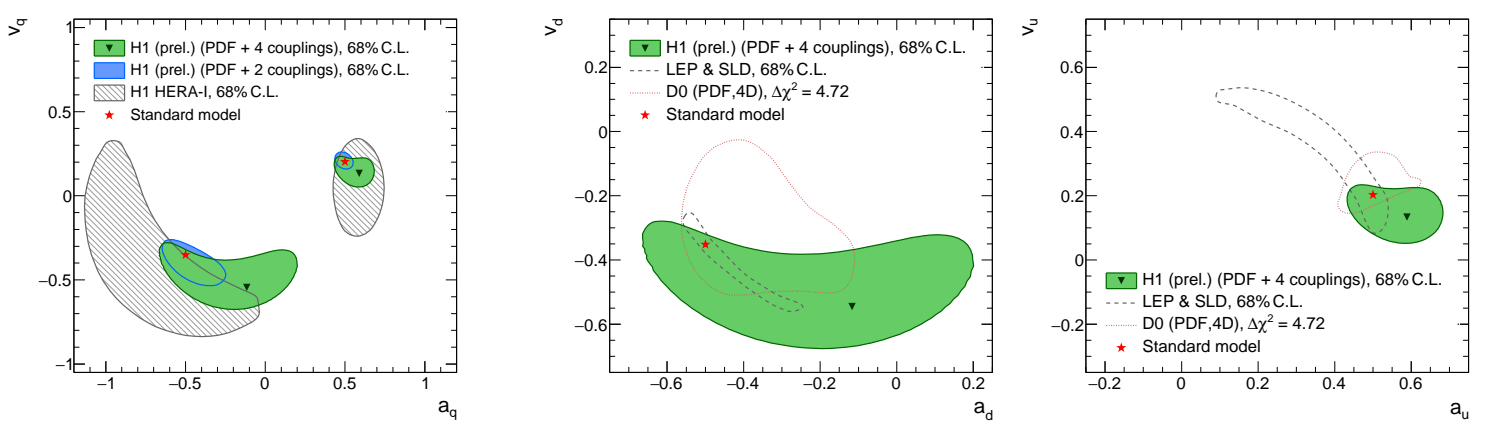

Figure 1: Left: results at $68 \%$ confidence level (C.L.) on the weak neutral current couplings of $u$ and $d$-type quarks to the $Z^{0}$-boson. The results are compared to previous results from H1 based on HERA-I data [3]. Middle and right: The results are compared with results from the LEP experiments and SLD [14], and D0 [15]. The mirror solutions of LEP are not shown. The displayed $68 \%$ C.L. contours of the H1 contours correspond to $\Delta \chi^{2}=2.3$, where all other fit parameters are minimised.

where the uncertainty represents the experimental and the PDF uncertainties, because these parameters are extracted simultaneously. Uncertainties on $m_{W}$ from the uncertainties on $m_{Z}$, the top-quark and Higgs-boson masses are comparably small. The inclusion of high- $Q^{2}$ HERA-II data significantly improves the precision of $m_{W}$ compared to an earlier extraction based in HERA-I data alone [3]. Fits of $G_{\mathrm{F}}$ and $\sin ^{2} \theta_{W}$ together with $m_{W}$ are displayed in fig. 2. For these fits, the quantities remain defined in the on-shell scheme, and the value of $m_{Z}$ is calculated (iteratively) from those parameters, $m_{W}$ and other boson and fermion masses which are input to the fits. The results are compatible with the best-known values of $G_{\mathrm{F}}$ and $\sin ^{2} \theta_{W}$ within a confidence level of $68 \%$.
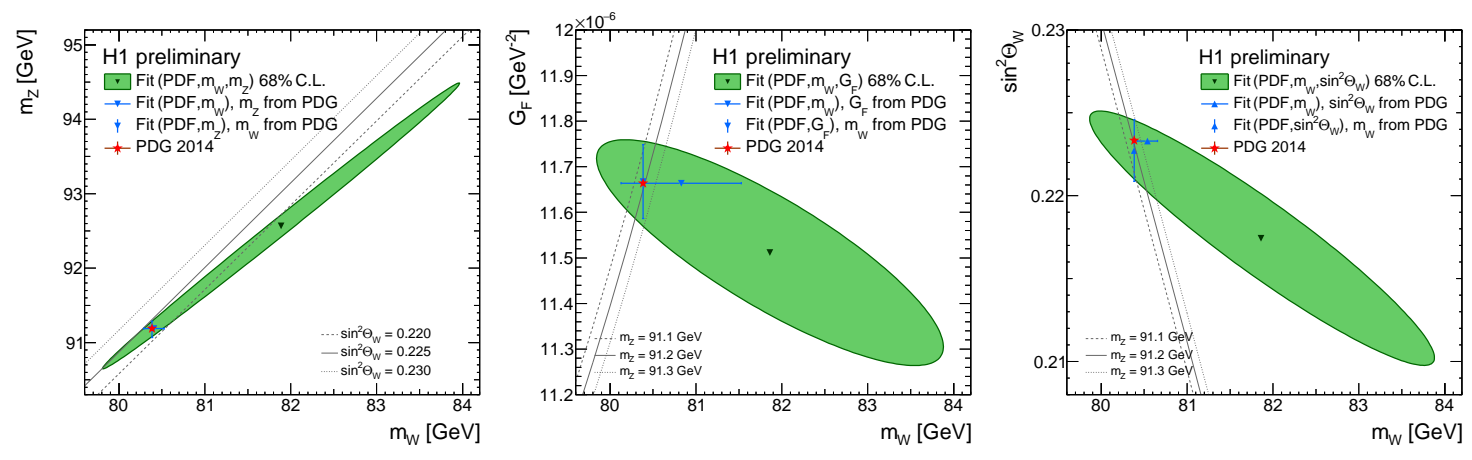

Figure 2: Fits of the PDF parameters and the $W^{ \pm}$-boson mass, together with the $Z^{0}$-boson mass, the Fermi Coupling Constant $G_{F}$ or the Weinberg angle $\sin ^{2} \Theta_{W}$. The respective calculations are all performed in the on-shell scheme, where $\alpha, m_{Z}$ and $m_{W}$ are the only electroweak parameters on Born-level. In case of the latter two fits, the value of $m_{Z}$ is calculated from the fit parameter and $\alpha, m_{W}$ and $\Delta r=\Delta r\left(\alpha, m_{W}, m_{Z}, m_{t}, m_{H}, \ldots\right)$.

The $Q^{2}$-dependence of the NC and CC data allows for a determination of the weak mixing angle at different scales. The data points are grouped into seven groups of data points with similar 
or identical values ${ }^{2}$ of $Q^{2}$. Each group is assigned an individual value of $\sin ^{2} \theta_{W}$, and a simultaneous fit of all values of $\sin ^{2} \theta_{W}$ and the PDF parameters are performed, where $m_{Z}$ is taken as external input parameter and the value of $m_{W}$ is calculated. The results are displayed in fig. 3. The extracted values of $\sin ^{2} \theta_{W}$ are consistent over the full $Q^{2}$-range with the best-known value [5], while the uncertainty is significantly higher.

\section{Summary}

A combined QCD and electroweak fit was performed to all published neutral and charged current scattering data by the H1 experiment. The inclusion of the cross section data from HERA-II with polarised lepton beams lead to a substantial improvement of the precision on the weak neutral couplings with respect to the previously published results based on the HERA-I data only. The results are complementary and competitive with those determined from other processes. The determination of parameters of the Standard Model, such

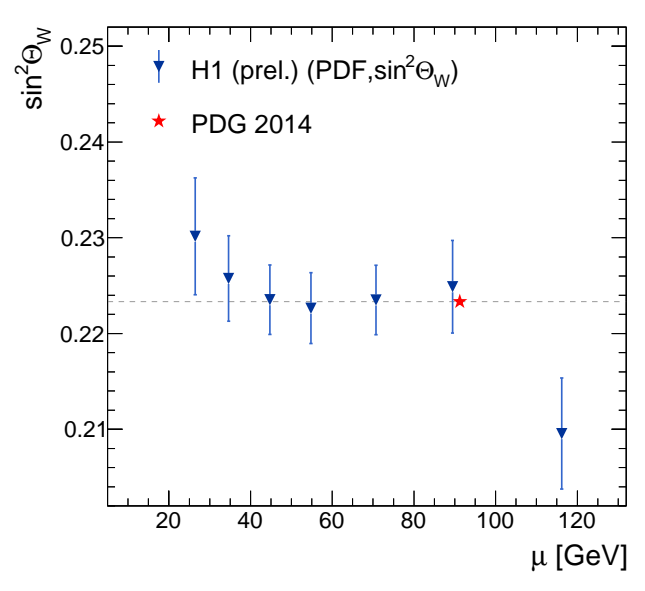
as the masses of the weak bosons, provide a complementary test to the direct measurements at the $Z$-pole and consistency with the bestknown parameters is found.

\section{References}

[1] H1 Collaboration, H1 preliminary report, H1prelim-16-041

[2] H. Abramowicz et al. [H1 and ZEUS Collaborations], Eur. Phys. J. C75 (2015) 12, [arXiv:1506.06042].

[3] A. Aktas et al. [H1 Collaboration], Phys. Lett. B 632, 35 (2006), [arXiv:hep-ex/0507080].

[4] H. Abramowicz et al. [ZEUS Collaboration], Phys. Rev. D 93 (2016) 092002.

[5] PDG Collaboration, K. A. Olive et al., Chin. Phys. C38 (2014) 090001.

[6] H. Spiesberger, "EPRC: A Program Package for Electroweak Physics at HERA", Contribution to the Workshop "Future Physics at HERA" (1996).

[7] M. Botje, Comput. Phys. Commun. 182 (2011) 490, [arXiv:1005.1481].

[8] F.D. Aaron et al. [H1 Collaboration], Eur. Phys. J. C71 (2011) 1579, [arXiv:1012.4355].

\footnotetext{
${ }^{2}$ Data points for $Q^{2} \leq 1200 \mathrm{GeV}^{2}$ and $Q^{2} \geq 12000 \mathrm{GeV}^{2}$ are grouped together, and otherwise all datapoints with an identical value of $Q^{2}$ are considered together.
} 
[9] C. Adloff et al. [H1 Collaboration], Eur. Phys. J. C13 (2000) 609, [hep-ex/9908059].

[10] C. Adloff et al. [H1 Collaboration], Eur. Phys. J. C19 (2001) 269, [hep-ex/0012052].

[11] C. Adloff et al. [H1 Collaboration], Eur. Phys. J. C30 (2003) 1, [hep-ex/0304003].

[12] C. Aaron et al. [H1 Collaboration], JHEP 1209 (2012) 061, [arXiv:1206.7007].

[13] V. Andreev et al. [H1 Collaboration], Eur.Phys.J.C75 (2015) 2, 65, [arXiv:1406.4709] .

[14] S. Schael et al. [ALEPH, DELPHI, L3, OPAL and SLD Collaborations], Phys. Rept. 427 (2006) 257, [hep-ex/0509008].

[15] V.M. Abazov et al. [D0 Collaboration], Phys.Rev. D84 (2011) 012007, [arXiv:1104.4590]. 